\title{
Revenue management based model for the dynamic definition of toll price for freight vehicles
}

\author{
S. Schreiner \& T. Hyodo \\ The Regional Planning Laboratory, \\ Tokyo University of Marine Science and Technology, Japan
}

\begin{abstract}
This study proposes a dynamic tolling method for a private toll network based on revenue management, taking into account the daily dynamics of freight transportation demand. The first part of this study assessed a sample of freight vehicles' routes, using maximum route overlapping methodology to obtain a relation between network attributes and the route choice of such vehicles. In the second part, also using route overlapping methodology, the individual value of time for each vehicle was estimated and incorporated to the route information as an attribute of the trip. The third part of this study uses the results obtained previously to model the dynamic tolling system. Discrete choice techniques are used to evaluate purchase probability during the day in relation to a set of possible toll prices, identifying the efficient prices that achieve revenue maximization. Finally, the model is tested by comparing the total daily toll revenue results of the current static price strategy and the dynamic pricing strategy. The data from the person trip survey conducted in 1999 in Tokyo is used as demand input data for the model.
\end{abstract}

Keywords: revenue management, toll road, route choice model.

\section{Introduction}

The long-term implications of the definition of a tolling scheme from the point of view of Revenue Management were introduced by Nagae and Akamatsu [1]. Although the focus of that work concentrated on showing that the long-term operation and day-to-day demand dynamics should be considered more dominant 
than the demand dynamics within a day, the study presented in this paper focus on the complementary objective of maximizing the expressway utilization by applying revenue management techniques to hourly control the toll fee.

The route choice is possibly the most critical issue when modeling a dynamic tolling system. The reason is certainly the heterogeneity of the demand and its variable perception of travel cost. This study focuses on the consideration of these both factors regarding freight transportation, which is far less affected by personal impressions of the way to be traveled than the passenger traffic. Even so, several attributes of the road network still affect the route choice. Schreiner et al [2] examined the influence of some of these attributes on the route choice of freight vehicles, showing that the influence of network attributes depends directly on the value of time. Based on that result, it is assumed that the user will take a route decision that considers exclusively the total perceived travel cost of each of the available routes. Naturally, it is also assumed that the user has knowledge of the network and its general level of service during the period when the trip takes place.

\section{Modelling methodology}

This study uses a Logit model to the calculation of the probability of a freight vehicle route use the tolled links. The utility is based on the generalized trip cost, which considers the influence of cognitive parameters in the length of the links and an empirical distribution of values of time. The obtained probability of using tolled links is then used in a revenue management model as single product case to estimate the demand rate according to a set of possible toll prices.

Let the set of links $\mathrm{L}=\left\{\mathrm{l}_{1}, \ldots, \mathrm{l}_{\mathrm{NOL}}\right\}$ and nodes $\mathrm{N}=\left\{\mathrm{n}_{1}, \ldots, \mathrm{n}_{\mathrm{NON}}\right\}$ define the network. Each link $l$ is represented by a vector of attributes such as length, number of lanes, lane width, type of link (tolled or non-tolled), toll price, etc.

The model is based on a discrete set of time intervals $t$, going from $t_{0}$ to $\mathrm{T}$. A trip is defined by the vector $r=\left\{\mathrm{O}_{\mathrm{r}}, \mathrm{D}_{\mathrm{r}}, \mathrm{VOT}_{\mathrm{r}}\right\}$. The vector includes the origin node, destination node, and individual value of time. The probability of arrival is given by $\lambda$. Let the path used by the demand route be defined as the set of links leading to the destination $\mathrm{D}_{\mathrm{r}}$, from the origin $\mathrm{O}_{\mathrm{r}}$, which incurs in the minimum total cost for the trip $r$, considering the cost for each link $l$ as

$$
G C_{l}=\left(\text { Fuel } \times \text { Eff } \times \text { Length }_{l}+V O T_{r} \times T T_{l}+\text { Toll }_{l}\right) \times \prod \beta_{k}^{z_{l k}}
$$

where:

$\mathrm{GC}_{1}$ : generalized cost of link $l$;

Fuel: fuel cost [JPY/liter];

Eff: engine efficiency [liters/km];

Length ${ }_{1}$ : lenth of link $l[\mathrm{~km}]$;

$\mathrm{VOT}_{\mathrm{r}}$ : value of time for the trip r [JPY/min];

$\mathrm{TT}_{1}$ : travel time on link $l$;

Toll 1 : Toll price on lin $\mathrm{k} l$.

$\mathrm{Z}_{\mathrm{lk}}$ is the $k$-th attribute (e.g. road width) for the $l$-th link of the $r$-th trip;

$\beta_{k}$ is an estimated parameter for the $k$-th attribute. 
The single product case is used in this model. The product is the use of tolled links for the trip $r$. The probability that the trip $r$ will include tolled links $\mathrm{P}$ (tolled) is obtained by considering the binary Logit model defined as

$$
P(\text { tolled })=\frac{e^{u_{\text {tolled }}}}{e^{u_{\text {tolled }}}+e^{u_{\text {non-tolled }}}}
$$

where the utility $\mathrm{u}$ is defined as

$$
u=-0.01 \times \operatorname{Cost}_{\text {path }}
$$

The Cost $\mathrm{path}_{\text {in }}$ is the total cost of the set of links used between one given origindestination pair. The trip cost is calculated as described in eqn. (1). For the computation of $u_{\text {tolled }}$, all tolled and non-tolled links are used. The computation of $\mathrm{u}_{\text {non-tolled }}$ considers exclusively non-tolled links. The possibility of non-travel was not considered.

For each time interval, the model considers the maximization of the toll revenue, based on the discrete demand probabilities of purchase. Let $p(t)$ denote the price at time interval $t$, which is in fact the price to be decided. Each price $\mathrm{p}(\mathrm{t})$ induces a unique demand rate $\mathrm{P}($ tolled). The price $\mathrm{p}(\mathrm{t})$ must be present in the set of allowable prices $P=\left\{\mathrm{p}_{\min }, \ldots, \mathrm{p}_{\max }\right\}$.

The demand rate $\mathrm{d}(\mathrm{t})$ is then defined as

$$
\mathrm{d}(\mathrm{t})=\lambda \mathrm{P}(\text { tolled })
$$

which represents the probability of demand at the time interval $t$ by a price $\mathrm{p}$. The revenue rate $\mathrm{r}(\mathrm{t})$ for a price $\mathrm{p}$ is then

$$
r(t)=p \times d(t)
$$

Given the discrete nature of the price set, the model identifies the efficient (revenue maximizing) prices to define the optimal price set.

Once the arrival probability and purchase probability are correctly computed, the definition of the efficient set of toll prices can be easily obtained.

\section{Data set and sample description}

The model is applied to the freight transportation demand identified by the 1999 Person Trip Survey in Tokyo Metropolitan Area. The network used in this case study is originally from the DRM (Digital Road Map) database, and is composed of 9,231 nodes and 25,062 links. The links present 25 attributes. From those, the following are used in this model: Length: length of the link (in meters); Toll1: a flag indicating whether the link is tolled or not; Toll2: a flag indicating whether the toll price of the link is "flat rate" or "length based"; Toll3: the toll price of the link; Assignment travel time: average travel time at the peak hour.

The case study network is presented in Figure 2, where the tolled links are highlighted in black. This area comprises different tolled networks with different toll price strategies. The model is applied exclusively to the access links of the Shuto-Expressway system, which uses a flat toll price scheme. The other expressway networks use different toll schemes, e.g. length based price, and were not included in the dynamic system. 


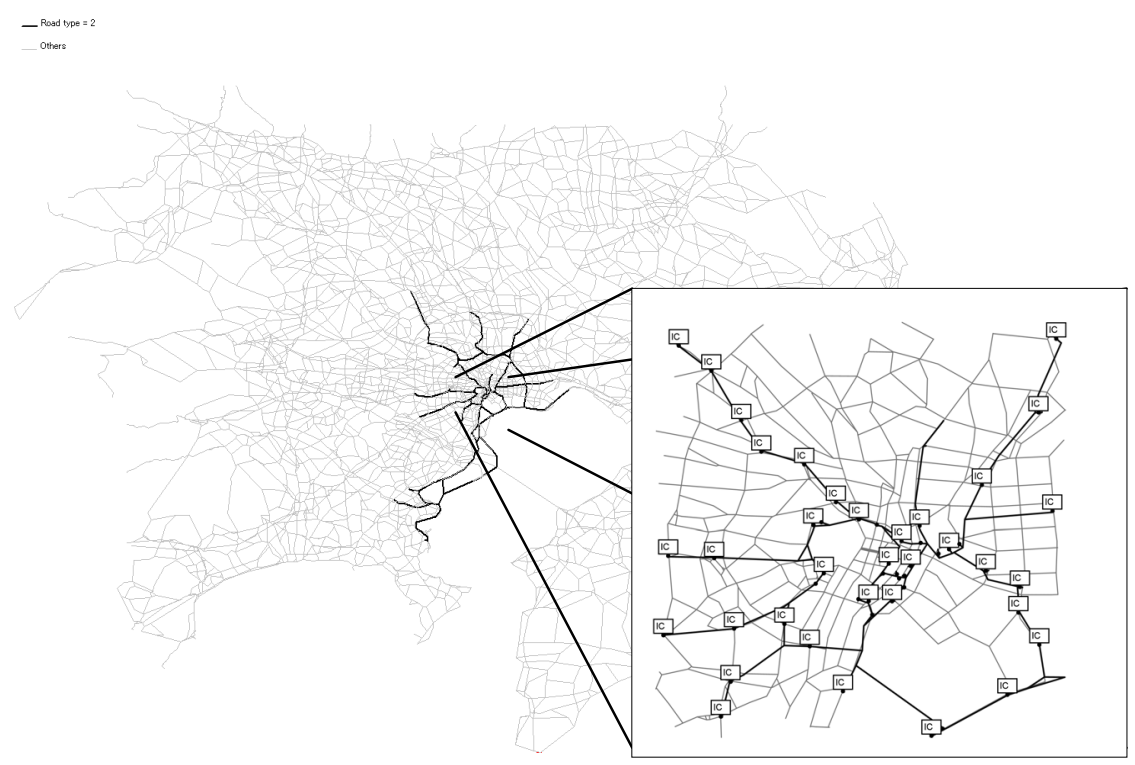

Figure 1: $\quad$ Tokyo metropolitan area network.

\subsection{Estimation of value of time}

Maximum route overlapping method was used for the estimation of the value of time (VOT). An important characteristic of this methodology is the possibility to estimate the VOT individually for each trip. The use of individual VOTs implies in a more detailed computation of individual choice behavior, as discussed by Schreiner [3].

This method is based on the maximization of the overlapping links between the calculated path and the observed path. This maximization is obtained through the variation of perception parameters which may be related to the network (e.g. number of lanes, restrictions to height and weight, etc.) or to the user (value of time, in this case).

These perception parameters are included in the model as link length modifying parameters [Eqn (6)] in the case of network attributes, and as generalized cost modifying parameter [Eqn (1)] in the case of the value of time.

The length modification is given by

$$
L_{a n}^{*}(\beta)=L_{a} \prod_{k} \beta_{k}^{z_{a k}}
$$

where:

$L_{a n}^{*}(\beta)$ is the perceived length of the a-th link at the $\mathrm{n}$-th trip sample;

$\mathrm{z}_{\mathrm{ak}}$ is the $\mathrm{k}$-th attribute (e.g. number of lanes) for the a-th link of the $\mathrm{n}$-th trip sample;

$\beta_{k}$ is the unknown parameter for the k-th attribute of the network. 
The objective function of the maximization procedure is defined as

$$
D(\beta, \omega)=\frac{\sum_{n} \sum_{a} \delta_{n a} \times \delta_{n a}^{*}(\beta, \omega) \times L_{a}}{\sum_{n} X_{n}}
$$

where:

$\delta_{n a}^{*}(\beta, \omega)$ : is equal to 1 if the $\mathrm{n}$-th trip's perceived minimum cost paths (for network parameters $\beta$ and value of time parameter $\omega$ ) include the a-th link, otherwise it is equal to 0 .

The maximization procedure considered values of time between 0 and 100 $\mathrm{JPY} / \mathrm{min}$. The results are shown in Figure 2.

In the model, these values of time were converted into trip attributes.

(Entire sample)

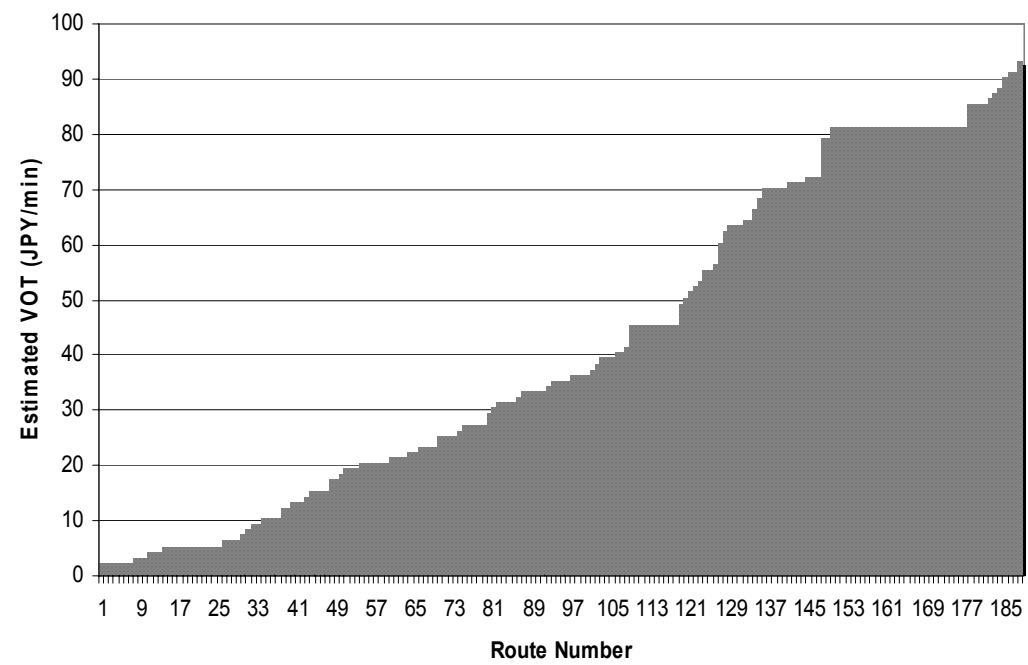

Figure 2: The estimated individual value of time.

The results in the modelling of value of time show a very heterogeneous distribution, indicating that a demand-wide generalization of value of time probably result in loss of accuracy on the route choice (specially whether use tolled routes or not).

\section{Model estimation results}

The probability of arrival is obtained from the analysis of the departure time of the trips surveyed in 1999. The model considers the peak hours volume as maximum possible probability of arrival and, proportionally, to the off-peak volumes. This distribution is presented in Fig. 3. 
The estimation of the probability of purchase requires the use of more detailed information, specifically the value of time distribution for the freight trips. This data is given by a set of freight vehicle trips that include the observed route. This set was used by Schreiner et al [2] to the evaluation of attributes influencing the route choice behavior, and, also, by Schreiner and Hyodo [3] to evaluate the individual value of time for each trip. The set of trips is composed by 597 observed routes of freight vehicles, including container-trailers and medium and small size trucks.

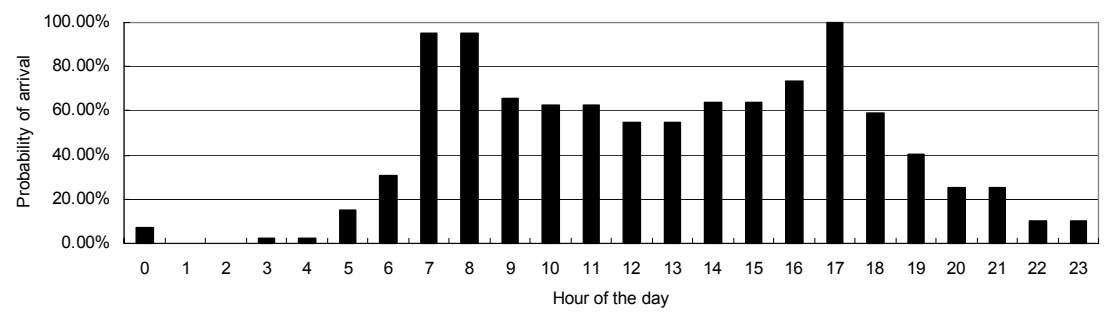

Figure 3: $\quad$ Estimated probability of arrival for tolled network demand in Tokyo by hour.

For each possible toll price $\mathrm{p}$, the probability of using tolled links $\mathrm{P}$ (tolled) was calculated for each trip and globally for the entire trip sample.

In the case study, the minimum and maximum toll price are set as $\mathrm{p}_{\min }=100$ JPY and $\mathrm{p}_{\max }=2,000 \mathrm{JPY}$. A practical increment of inc $=100 \mathrm{JPY}$ is used. Figure 4 shows the obtained results for the global $\mathrm{P}($ tolled $)$.

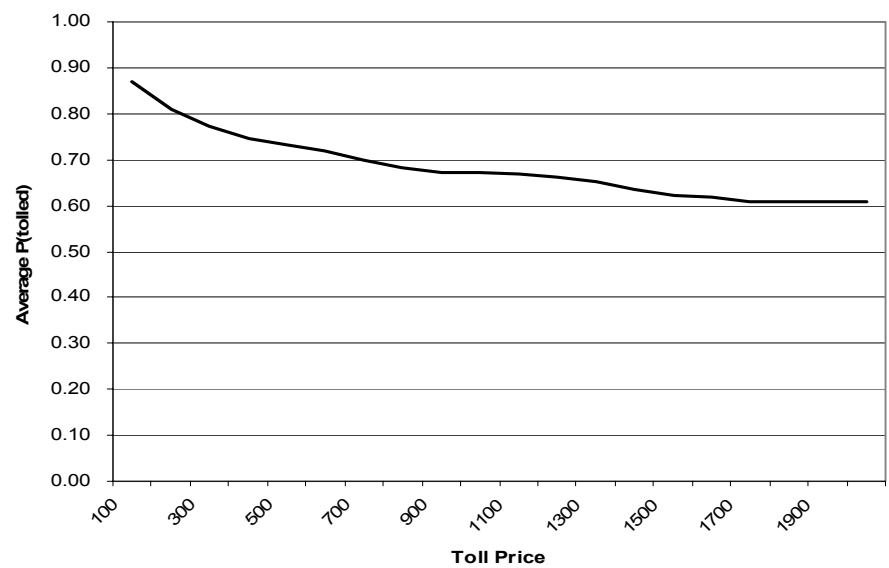

Figure 4: Average $\mathrm{P}($ tolled) in relation with the toll price for the entire trip sample. 
The results show an stabilization of $\mathrm{P}($ tolled) around $62 \%$ for toll prices over JPY 1,700 . This behavior was caused by the migration of the demand to the other tolled networks (where the RM model was not applied).

The demand rate $\mathrm{d}(\mathrm{t})$ with relation to each possible toll price, calculated based on the sample of freight vehicle trips, is shown in Figure 5.

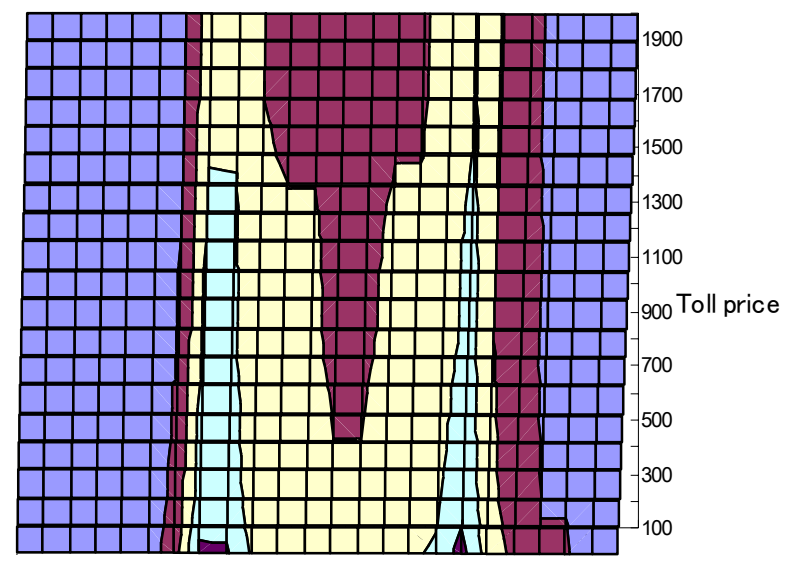

Hour of the day

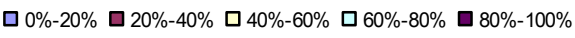

Figure 5: Demand rate in relation with the price and hour of the day.

Similar to the definition of portfolio investment composition used in financial economics, the key factor in the decision of the toll price is the respective risk, in this case, with respect to the demand. Taking in consideration that the current toll price is JPY 1,400 for large vehicles, it is possible assess the current demand rate, e.g., between $8 \mathrm{a} . \mathrm{m}$. and 9a.m. the demand rate is currently in the interval 60 to $80 \%$. The tactical advantage of the use of revenue management for the definition of the toll price can be observed in the hypothetical situation of increasing the toll price in this same time interval (8a.m. to 9a.m.) by JPY 100. The new price (JPY 1,500) would take the demand rate to the next lower interval of $40 \%$ to $60 \%$, possibly resulting in reduction of revenue on the long run.

\section{Model results}

The demand rate estimations presented in the previous section permit the comparison, in terms of revenue performance, between the current toll price scheme and a hypothetical new price scheme.

The normal toll price for freight vehicles in the Tokyo Expressway System is JPY 1,400 (approximately 14.00 USD). The company operating the system has 
introduced some discount schemes including even the peak time. Currently, the toll prices are as presented in Table 1 (Source: Metropolitan Expressway Co., Ltd. Web-site). Additional discounts are eventually offered in special occasions, such as prolonged holidays, however they are not considered here.

Table 1: $\quad$ Current price scheme in Tokyo expressway system.

\begin{tabular}{c|c|c}
\hline Normal & all day & 1400 JPY (aprox 14 USD) \\
\hline \multirow{2}{*}{ Peak discount } & $6 a m-11 \mathrm{am}$ & \multirow{2}{*}{$1360 \mathrm{JPY}$ (aprox 13.60 USD) } \\
\cline { 2 - 2 } & $3 \mathrm{pm}-6 \mathrm{pm}$ & \\
\hline \multirow{2}{*}{ Off peak discount } & $11 \mathrm{am}-3 \mathrm{pm}$ & \multirow{2}{*}{$1260 \mathrm{JPY}$ (aprox 12.60 USD) } \\
\cline { 2 - 2 } & $6 \mathrm{pm}-10 \mathrm{pm}$ & \\
\hline Night discount & $10 \mathrm{pm}-6 \mathrm{am}$ & $1120 \mathrm{JPY}$ (aprox 11.20 USD) \\
\hline
\end{tabular}

In order to perform the comparative analysis of the revenue performance of both schemes, the alternative prices presented in Table 2 were used. This price scheme represents the toll price that induces a demand rate $1 \%$ over the demand rate of the current price scheme.

Table 2: $\quad$ Alternative prices based on the model results.

\begin{tabular}{c|c|c}
\hline \multirow{2}{*}{ Peak discount } & $6 a m-11 \mathrm{am}$ & \multirow{2}{*}{$1280 \mathrm{JPY}$ (aprox 12.80 USD) } \\
\cline { 2 - 2 } Off peak discount & $3 \mathrm{pm}-6 \mathrm{pm}$ & \\
\cline { 2 - 2 } & $11 \mathrm{am}-3 \mathrm{pm}$ & \multirow{2}{*}{$1100 \mathrm{JPY}$ (aprox 11.00 USD) } \\
\hline Night discount & $10 \mathrm{pm}-10 \mathrm{pm}$ & \\
\hline
\end{tabular}

The difference in total revenue at the interchanges where the new toll price scheme was implemented is presented in Table 3.

Table 3: $\quad$ Results of model implementation.

\begin{tabular}{c|c|c|}
\hline Operations hours & Revenue & Veh.km \\
\hline Peak discount & $+2.82 \%$ & $+0.52 \%$ \\
\hline Off peak discount & $-0.84 \%$ & $+1.94 \%$ \\
\hline Night discount & $-25.57 \%$ & $+2.96 \%$ \\
\hline
\end{tabular}

The results showed positive revenue modifications exclusively in the peak time period, when the increase in traffic using the interchanges within the study area compensate the reduction in the marginal revenue. For the off-peak and night time periods, the reduction in the marginal revenue had a major impact in the total revenue.

More detailed analyses of these results show a significant variation of revenue results within each time period, which suggests that the revenue management 
model will produce better results if applied to a different timetable with shorter time periods.

\section{Conclusions}

The analyses permit to reach conclusions on the issues: potentiality and applicability of the model; impacts in revenue and traffic volume; and recommendations for further research.

The model showed a potential use for revenue management in the maximization of toll revenue. The requirements in terms of data management, which historically impose a barrier for revenue management implementation, are now available in several cities through electronic toll collection.

The proposed model showed positive results in increasing the total revenue during the peak time period. During other periods the model resulted in considerably decrease in total revenue. Based on the behaviour of the demand rate, it is concluded that a price-time framework with shorter intervals would improve the efficiency of the price scheme.

The changes in departure time are considered in the dynamic stochastic assignment method used for the model testing as temporal utility; however they are considered in the proposed model. As next step, it is recommended that the impact in departure time be included in the modelling process, which incurs in the development of a detailed assignment algorithm that considers variations in the value of time at OD pair level and variations in temporal utility.

The assessment of the impact of demand heterogeneity was possible through using average values of value of time for a larger number of demand subsegments within the freight vehicle segment. It is recommended that in future data collections, the type of truck and type of cargo are also included, in order to possibly include these parameters in the model.

Finally, it was concluded that a variable toll price system may optimize the revenue collection, depending on the correct definition of the intervals for price change and definition of more specific vehicle classes for toll pricing.

\section{References}

[1] Nagae, T. \& Akamatsu, T., Dynamic Revenue Management of a Toll Road Project under Transportation Demand Uncertainty, Networks and Spatial Economics Vol.6, pp.345-357, 2006.

[2] Schreiner, S., Hyodo, T. \& Takahashi, Y., A Study on the Influence of Network Attributes on the Route Choice Behavior, Eastern Asia Society of Transportation Studies, Vol. 7, pp. 642-653, 2007.

[3] Schreiner, S. \& Hyodo, T., A Study on the Estimation of Value of Time based on Truck Route Samples and Its Applications in Assignment Procedures, Japan Society of Traffic Engineers, Proceedings of the $27^{\text {th }}$ Annual Meeting, 2007. 